\title{
Core-Shell Nanostars for Multimodal Therapy and Imaging
}

\author{
Mengyuan Li, Lele Li, Changyou Zhan, Daniel S. Kohane ${ }^{\bowtie}$ \\ Laboratory for Biomaterials and Drug Delivery, Department of Anesthesiology, Division of Critical Care Medicine, Children's Hospital Boston, Harvard Medical \\ School, Boston, MA, 02115. Department of Chemical Engineering, Massachusetts Institute of Technology, Cambridge, MA, 02139. Koch Institute for Integrative \\ Cancer Research, Massachusetts Institute of Technology, Cambridge MA 02139, USA. \\ $\triangle$ Corresponding author: daniel.kohane@childrens.harvard.edu.
}

() Ivyspring International Publisher. Reproduction is permitted for personal, noncommercial use, provided that the article is in whole, unmodified, and properly cited. See http://ivyspring.com/terms for terms and conditions.

Received: 2016.04.13; Accepted: 2016.06.16; Published: 2016.10.01

\begin{abstract}
The coupling of diagnostic capability and effective therapy in a single multifunctional nanomedicine is desirable but remains challenging. Here, we developed multifunctional nanoparticles consisting of a gold nanostar (AuNS) core with a shell of metal-drug coordination polymer (CP). The AuNS core enabled plasmonic photothermal effect and two-photon photoluminescence (TPL), while the $\mathrm{CP}$ shell of gadolinium and gemcitabine monophosphate allowed chemotherapy and MRI imaging. The AuNS@CP nanoparticles exhibited a strong $T_{1}$ contrast signal and could monitor the localization of nanoparticles in vivo through noninvasive MR imaging, while intravital TPL imaging could be used to study nanoparticle behavior in tumors at the microscopic level. The combination of photothermal therapy and chemotherapy inhibited tumor growth in vivo.
\end{abstract}

Key words: gold nanostar, imaging

\section{Introduction}

Nanoparticles have been used to deliver a wide range of therapeutics for cancer treatment. A presumed cornerstone of such treatment is the tendency of nanoparticles to accumulate in tumors due to their leakier vasculature and impaired lymphatics, a phenomenon termed enhanced permeability and retention (EPR) [1-3]. However, the EPR effect is variable from patient to patient, which may affect individual therapeutic effectiveness [4]. Consequently, there has been interest in integrating imaging and therapy in a single theranostic nanoplatform to monitor its accumulation and efficacy noninvasively, allowing adjustment of a regimen to the patient's specific responses to treatment [5-10].

Gold nanoparticles (AuNPs), such as nanorods, nanocages, and nanostars can absorb near-infrared (NIR) light and transduce photon energy into heat for the thermal ablation of tumors because of surface plasmon resonance (SPR) [11-21]. Since NIR light can penetrate releatively deeply into tissue without causing damage to healthy tissues, such photothermal therapy (PTT) is noninvasive and highly localized, with minimal systemic toxicity [11-18]. However, PTT alone may be unsuccessful in eradicating cancer cells completely because of uneven heat distribution and attenuation of the light intensity as it travels deeper into tissue [14-16]. The light-to-heat conversion efficiency of AuNPs may also be changed by internalization in cancer cells [19]. The combination of PTT with chemotherapy within a single multifunctional nanoconstruct has been proposed to overcome these limitations [16]. However, most anticancer drugs cannot be absorbed onto naked AuNPs surfaces due to their weak interaction with $\mathrm{Au}$ [16]. The strategies reported for drug confinement on or around AuNPs often require complex surface coatings of AuNPs (e.g., mesoporous silica, polymer) through multiple steps [22, 23]. A simple and efficient strategy for the integration of imaging and 
therapeutic agents in a single AuNP is desirable for combined therapy (PTT + chemotherapy) to improve local therapeutic efficacy, and for imaging to monitor the delivery and distribution of nanoparticles [16].

Here we report a strategy to coat the AuNP surface with a metal-drug coordination polymer (CPs) (Fig. 1). CPs, including metal-organic frameworks (MOFs), are a new class of hybrid materials composed of metal ions and organic ligands linked by coordination bonds. [24-27] At the nanoscale, CPs are promising materials for vehicles to deliver imaging agents and therapeutics, due to their compositional and structural tunability, high capacity for drug loading, metal sites accessible for binding by functional groups on drugs, and ease of surface functionalization. [27-31] We report the synthesis of core-shell nanocomposites consisting of a gold nanostar (AuNS) as the core and a shell of metal-drug CP (Gd(III) and gemcitabine phosphate). AuNS with multiple branches were chosen here because of their easy synthesis without cytotoxic surfactants and the potential for high drug loading due to their high surface-to-volume ratio [18-21]. Furthermore, the plasmon resonance spectra of AuNS can be precisely tuned by adjusting their length or number of spikes [19]. This system (AuNS@CP) was designed to combine photothermal therapy and chemotherapy with magnetic resonance (MR) for clinical imaging, as well as two-photon luminescence (TPL) imaging to enable research on nanoparticle behavior in vivo. We applied the AuNS@CP for imaging and cancer therapy in a breast cancer xenograft model using the $4 \mathrm{~T} 1$ cell line, which is sensitive to gemcitabine [32].

\section{Results and Discussion}

\section{Preparation and Characterization of AuNS@CP}

The CP shell was composed of the anti-cancer drug gemcitabine-5'-monophosphate (GMP, see Fig. S1 for the structure) and Gd(III), a coordination complex we have reported recently [33]. Gd(III) was used because of its coordination flexibility and its function as an MRI contrast agent [34]. The core-shell AuNS@CP nanostructures were synthesized by a two-step method. First, AuNSs (Fig. 2A, B) with a mean diameter of $46 \mathrm{~nm}$ (by dynamic light scattering, DLS, Fig. S2) were synthesized by the reduction of an $\mathrm{Au}$ (III) salt precursor in HEPES buffer [35]. To ensure that the CPs formed a coating on the AuNS (rather than forming particles by self-nucleation) [31], the particles were first incubated with GMP to allow its absorption by coordination interaction between the cytosine moiety of GMP and the gold surface [30,31]. Then the GMP-coated AuNS were mixed in an aqueous solution of $\mathrm{GdNO}_{3} \cdot 3 \mathrm{H}_{2} \mathrm{O}$ to promote the growth of GMP/Gd CP on the surface of the AuNS. The particle surface was PEGylated with methoxy-PEG bearing two phosphate groups at one terminus (PEG-DiP, see Fig. S1 for the structure) that could coordinate with Gd(III). The PEGylation of nanoparticles was confirmed by Fourier transform infrared (FTIR) spectroscopy (Fig. S3), which showed the $\mathrm{C}-\mathrm{O}-\mathrm{C}$ stretching vibrations band at $1095 \mathrm{~cm}^{-1}$ and $\mathrm{C}-\mathrm{H}$ bending vibrations band at $1336 \mathrm{~cm}^{-1}$, which are characteristic of PEG [33, 38].

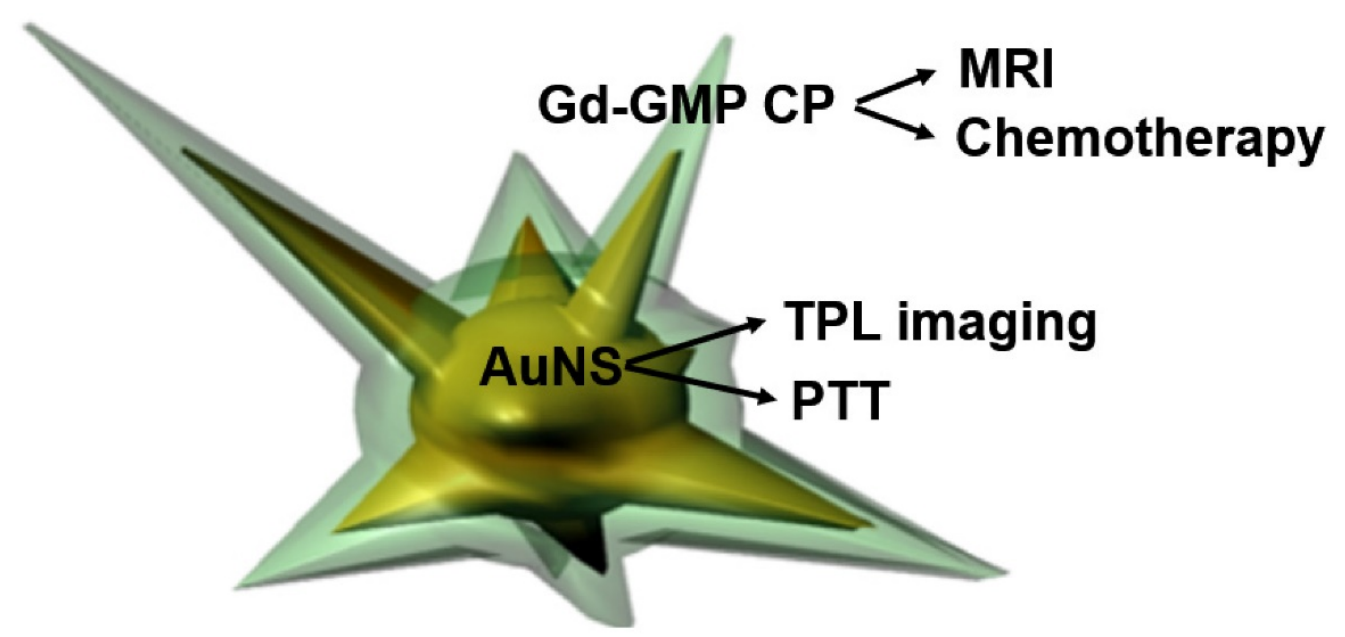

AuNS@CP

Figure 1. Schematic illustration of the core-shell AuNS@CP nanostructure. 

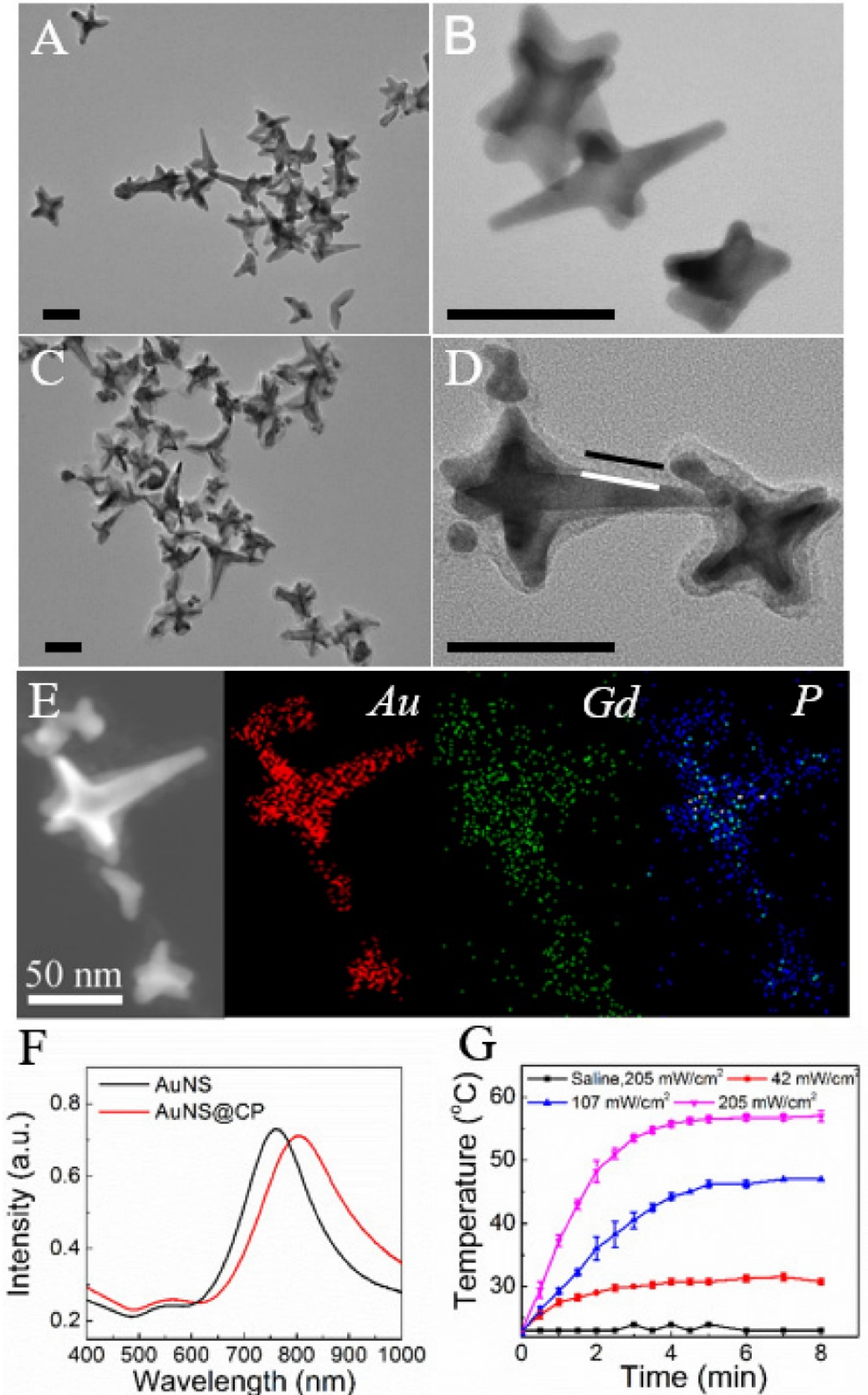

Figure 2. Characterization of core-shell AuNS@CP nanostructures. (A-D) TEM images of (A,B) AuNS and (C,D) AuNS@CP (space between black and white lines in $D$ indicates CP layer). Scale bar: $50 \mathrm{~nm}$. (E) HAADF-STEM image of AuNS@CP and corresponding EDX elemental maps. (F) UV-vis-NIR absorbance spectra of AuNS and AuNS@CP nanoparticles. (G) Effect of irradiance and duration of irradiation (808 nm continuous wave NIR laser) on the temperature of a solution of AuNS@CP nanoparticles. Data are means \pm SD $(N=4)$.

The particles had a well-defined core-shell structure. The shell thickness could be modified from $1.8 \pm 0.8$ to $7.2 \pm 3.1$ by increasing the mass ratio of GMP and $\mathrm{GdNO}_{3} \cdot 3 \mathrm{H}_{2} \mathrm{O}$ from 1.5:0.6 to 4:1.6, while the mass ratios of AuNS and PEG-DiP were kept constant (3:2) (Fig. S4). For downstream experiments we used particles with shell thicknesses of $5.1 \pm 1.8 \mathrm{~nm}$, because they allowed the best balance of drug loading without aggregation during synthesis. Elemental analysis by energy-dispersive X-ray spectroscopy (EDX) confirmed the presence of gold (from AuNS), fluorine and phosphorus (from GMP), and Gd in the particles (Fig. S5). The composition of the core-shell nanostructure was confirmed by the EDX elemental mapping images obtained from high angle annular dark field (HAADF) scanning transmission electron microscopy (STEM) (Fig. 2E), which showed the presence of the elements $\mathrm{Au}, \mathrm{Gd}$, and $\mathrm{P}$ (in GMP) in the nanostructures. AuNS@CP remained monodisperse without obvious aggregation for $24 \mathrm{~h}$ in human serum buffer (human serum : saline $=1: 1, \mathrm{v} / \mathrm{v}$, $\mathrm{pH}$ 7.4) (Fig. S6; compare to Fig. 2c). UV-vis 
spectroscopy of as-synthesized AuNS showed maximal absorption at $760 \mathrm{~nm}$ (Fig. 2F). A red shift to $805 \mathrm{~nm}$ occurred upon formation of the core-shell structure.

The proportions of $\mathrm{Au}, \mathrm{GMP}$ and $\mathrm{Gd}(\mathrm{III})$ in the AuNS@CP were 36.8 wt $\%, 31.5$ wt $\%$, and $13.2 \mathrm{wt} \%$, respectively, as determined by HPLC and inductively coupled plasma mass spectrometry (ICP-MS) (see SI for method of calculation). The loading efficiencies of GMP and Gd (III) were $85.7 \%$ and $90.0 \%$, respectively. GMP release studies were performed by immersing a dialysis tube filled with $1.0 \mathrm{~mL}$ of AuNS@CP (2.5 mM $\mathrm{Au}$ in saline) into $13.5 \mathrm{~mL}$ of saline. At predetermined time intervals, $1 \mathrm{~mL}$ of the external saline was removed (and replaced) and the amount of released GMP was analyzed by HPLC. Approximately $49 \%$ of GMP was released from AuNS@CP in 24 h (Fig. S7).

The photothermal capability of AuNS@CP (0.5 $\mathrm{mM} \mathrm{Au}$ in saline) was investigated by irradiating them with an $808 \mathrm{~nm}$ continuous wave NIR laser (Fig. $2 \mathrm{G}$ ). The bulk temperature of the aqueous solution was measured with an infrared thermal camera. Irradiation caused the temperature of the AuNS@CP solution to increase rapidly during the first 2-4 $\mathrm{min}$ and plateau within $5 \mathrm{~min}$. The plateau temperature increased from 31 to $57^{\circ} \mathrm{C}$ as the irradiance increased from 42 to $205 \mathrm{~mW} / \mathrm{cm}^{2}$. The temperature increase was also dependent on the AuNS@CP concentration (Figure S8). The plateau temperature increased from 33 to $56^{\circ} \mathrm{C}$ as the $\mathrm{Au}$ concentration increased from 0.0625 to $0.25 \mathrm{mM}$ with light irradiation $\left(0.5 \mathrm{~W} / \mathrm{cm}^{2}\right)$.
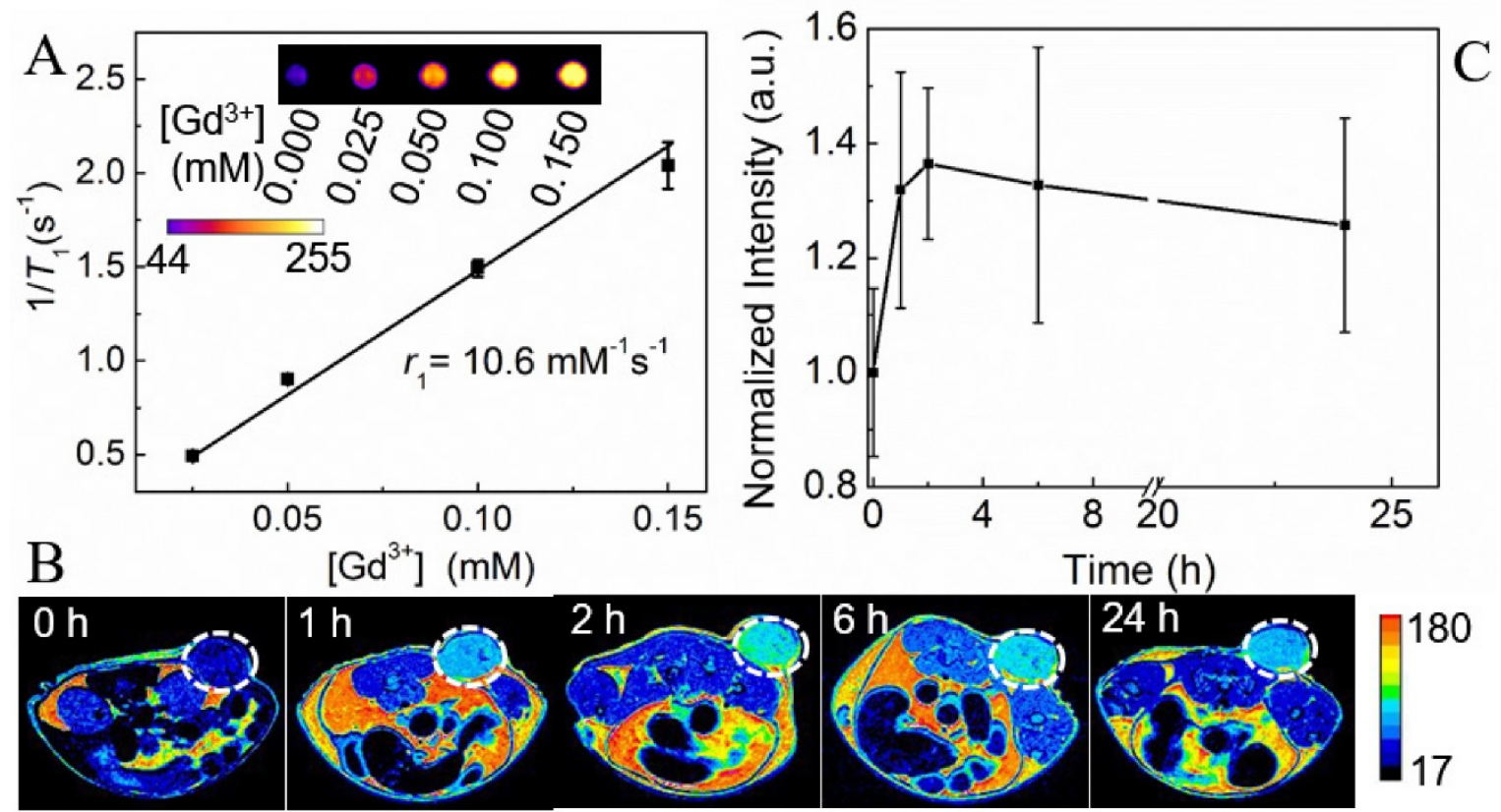

The temperature change in irradiated saline without AuNS@CP was negligible.

\section{MR Imaging with AuNS@CP}

The $T_{1}$-weighted MRI signals of tubes containing a range of concentrations of aqueous AuNS@CP were measured with a 7T MRI scanner. $T_{1}$-weighted MR images (Fig. 3A, inset) showed that the $T_{1}$-weighted MRI signal intensity increased with increasing concentration of AuNS@CP. The molar relaxivity $\left(r_{1}\right)$ of AuNS@CP, an important parameter that determines the efficiency of a MRI contrast agent, was determined to be $10.6 \mathrm{mM}^{-1} \cdot \mathrm{s}^{-1}$ by calculating the slope of the line relating the $\mathrm{Gd}(\mathrm{III})$ concentration to the longitudinal relaxation rate $\left(1 / T_{1}\right)$ of water protons (Fig. 3A). To examine the performance of AuNS@CP as MRI contrast agents in vivo, nude mice bearing $\sim 200 \mathrm{~mm}^{3}$ subcutaneous $4 \mathrm{~T} 1$ breast cancer cell tumors were administered intravenous AuNS@CP (0.05 mmol $/ \mathrm{kg} \mathrm{Gd}(\mathrm{III}))$. $T_{1}$-weighted MR images were acquired before injection and at predetermined time points afterwards. Enhancement of $T_{1}$-weighted MR signal intensity in the tumor was noted after injection compared with pre-injection values: 1.29 -fold at $1 \mathrm{~h}$, 1.39-fold at $2 \mathrm{~h}, 1.43$-fold at $6 \mathrm{~h}$ and the enhancement remained at 1.24-fold $24 \mathrm{~h}$ post-injection (Fig. 3B, C). This in vivo MR imaging efficacy and prolonged MRI contrast enhancement of the tumor are comparable with those of other Gd-based NPs reported recently $[38,39]$.

Figure 3. MR imaging of AuNS@CP in vitro and in vivo. (A) Plot of longitudinal relaxation rate $\left(1 / T_{1}\right)$ as a function of Gd(III)-concentration in AuNS@CP nanoparticles. The slope indicates the molar relaxivity $\left(r_{1}\right)$. Data are means \pm SD $(N=4)$. Inset: Color-coded $T_{1}$-weighted MR images of tubes containing AuNS@CP nanoparticles at different Gd(III) concentrations, from which the data in the graph were derived. (B) In vivo $T_{1}$-weighted MR images (color-coded by intensity) acquired before and at different time points after i.v. injection of AuNS@CP nanoparticles in 4T1 tumor-bearing mice. Tumors are indicated by white dashed circles. (C) Change in the MRI signal intensity of tumor sites after injection of AuNS@CP nanoparticles. Data are means \pm SD (N=4) of the quantification of the data in panel (B). 


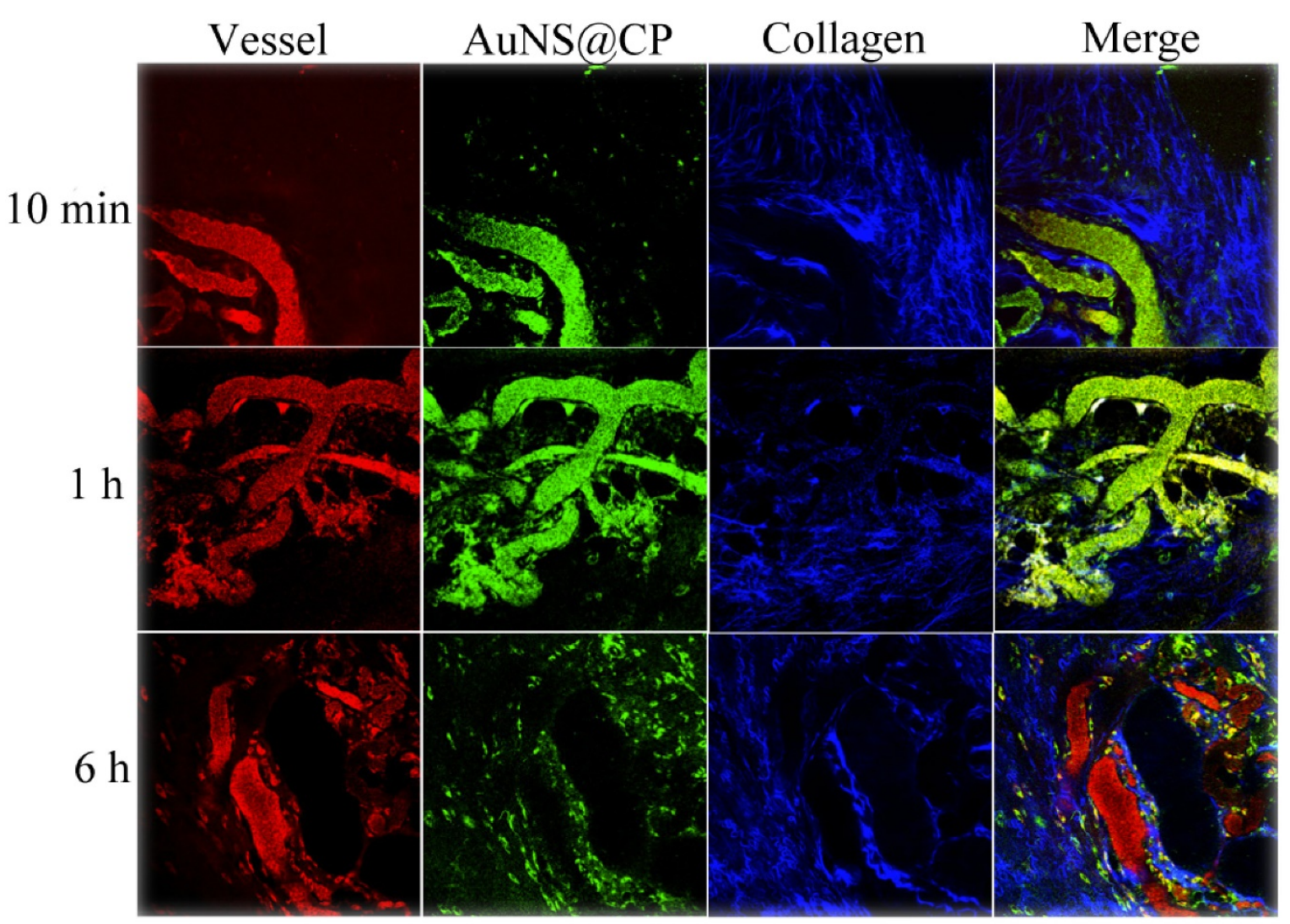

Figure 4. Representative TPL intravital microscopic images of AuNS@CP nanoparticles in orthotopic 4T1 tumors after i.v. administration of AuNS@CP nanoparticles. Green: AuNS@CP, red: Texas Red-dextran (70 kDa) that demarcates blood vessels, blue: intratumoral collagen matrix, yellow: (in the Merge panel), the localization of AuNS@CP in blood vessels.

\section{TPL Imaging of Tumor Microenvironment}

In two-photon luminescence (TPL), two low energy photons excite a fluorophore with subsequent emission of a fluorescence photon of higher energy. [40] TPL is noninvasive and can penetrate deeply in tissue because much of the emitted light is in the NIR region [40-41]. Due to its high resolution, TPL can be a useful research tool for imaging and tracking at the microscopic level [40-43] (MRI, CT, and PET have lower resolution and are often used for whole body imaging). In general, gold nanostructures, such as AuNS, can provide stronger TPL signals than organic fluorophores [42, 43]. We used real-time intravital TPL imaging to probe the distribution of AuNS@CP in the tumor microenvironment.

Nude mice bearing $\sim 200 \mathrm{~mm}^{3}$ subcutaneous $4 \mathrm{~T} 1$ breast cancer tumors were injected intravenously with AuNS@CP (250 nmol Au/kg). Texas Red-dextran (molecular weight $70 \mathrm{kDa}$ ) was also injected to highlight the vasculature [44]. Immediately following injection of Texas Red-dextran, the mice were anesthetized and the tumor was exposed by a skin incision. The tumor was then immobilized on the microscope stage for TPL imaging (with NIR excitation of $810 \mathrm{~nm}$ ), while continuity with the body vasculature was maintained. [45] Intravital multiphoton microscopy imaging of the tumor (Fig. 4) revealed that the AuNS@CP reached the tumor microvasculature by $10 \mathrm{~min}$ after intravenous injection. By 1 hour postinjection, extravasated photoluminescence (indicating AuNS@CP) was observed, and was still present 6 hours after injection. Tumor uptake of the NPs was further confirmed by 3D reconstruction of the TPL imaging data of the tumor (Fig. S9), showing that the AuNS@CP nanoparticles were mainly distributed outside the blood vessels 6 hours after injection.

\section{Combined Photothermal-chemotherapy}

The photothermal and chemotherapeutic effects of the AuNS@CP were evaluated in vitro and in vivo with 4T1 breast cancer cells. In vitro, AuNS@CP and free GMP exhibited similar time- and concentration-dependent cytotoxicity at equivalent GMP concentrations over 48 hours (Fig. S10). AuNS alone showed little cytotoxicity at a wide range of concentrations that are comparable to concentrations one might expect in vivo (Fig. S11) [11, 12]. Irradiation decreased the viability of $4 \mathrm{~T} 1$ cancer cells incubated with AuNS (Au dose: $11.7 \mu \mathrm{g} / \mathrm{mL}$ ) (Fig. 5). Irradiation of cells incubated with AuNS@CP (GMP: $10.0 \mu \mathrm{g} / \mathrm{mL}$ ) 
containing the same concentration of gold further reduced survival to $3 \%$. Similar degrees of cell killing could also be achieved by irradiating cells incubated with a higher concentration of AuNS alone (Au: 23.4 $\mu \mathrm{g} / \mathrm{mL}$; Fig. S12), demonstrating that enhanced cytotoxicity could be realized with lower doses of AuNS@CP nanoparticle by the addition of chemotherapy. Irradiation itself had no effect on cell survival, alone or incubated with equivalent concentrations of GMP (Fig. 5). We also tested cell viability as a function of AuNS@CP concentration with and without irradiation $\left(0.5 \mathrm{~W} / \mathrm{cm}^{2}\right)$ (Figure S13). AuNS@CP could produce significant photothermal ablation (beyond that provided by the particles alone) at Au concentrations higher than 2.3 $\mu \mathrm{g} / \mathrm{mL}$ (GMP: $2.0 \mu \mathrm{g} / \mathrm{mL}, 5 \mu \mathrm{M}$ ). Maximal killing ( $96 \%$ of cells were killed) by irradiating particles was achieved at a $\mathrm{Au}$ concentration of $7.05 \mu \mathrm{g} / \mathrm{mL}$ (GMP: $6.0 \mu \mathrm{g} / \mathrm{mL}, 15 \mu \mathrm{M})$.

The effectiveness of AuNS@CP was investigated in vivo in female $4 \mathrm{~T} 1$ breast tumor-bearing mice. When tumors reached about $50 \mathrm{~mm}^{3}$, mice were given an intravenous injection of $200 \mu \mathrm{L}$ of one of the following: saline, GMP (10.3 mg GMP/kg), AuNS (12.0 mg Au/ kg), a mixture of free GMP and AuNS (termed AuNS + GMP; $10.3 \mathrm{mg}$ GMP $/ \mathrm{kg}$ separate from $12.0 \mathrm{mg} \mathrm{Au} / \mathrm{kg}$ ), or AuNS@CP (with $10.3 \mathrm{mg}$ $\mathrm{GMP} / \mathrm{kg}$ bound to $12.0 \mathrm{mg} \mathrm{Au} / \mathrm{kg}$ ). One group received PEGylated AuNS to control for the effect of irradiation of gold alone; their absorbance peak at 804nm (Fig S14) shows their absorptive properties were similar to those of AuNS@CP (Fig. 2F). In the laser-treated groups, the tumor site was irradiated with a NIR laser $\left(808 \mathrm{~nm}, 0.5 \mathrm{~W} / \mathrm{cm}^{2}\right)$ for 3 minutes, 6 hours after intravenous injection. In the AuNS@CP + laser group, the temperature of the tumor area, recorded by an infrared thermal camera (Fig. 6A, B), reached $53{ }^{\circ} \mathrm{C}$ after $3 \mathrm{~min}$ of irradiation, which is sufficient to damage cancer cells irreversibly [39]. The same region reached $39^{\circ} \mathrm{C}$ in irradiated saline-treated animals (Fig. 6A, B).

Mice treated with saline exhibited rapid tumor growth (Fig. 6C), with a mean tumor volume of 1145 $\mathrm{mm}^{3}$ on day 15 . There was no statistically significant difference in final tumor size between saline and saline + laser treated groups $(p>0.1)$, indicating that laser irradiation alone had no effect on tumor growth. Mice treated with free drug showed a slightly delay in tumor growth, with a median tumor volume of 876 $\mathrm{mm}^{3}$ on day 15 ( $\mathrm{p}=0.008$ vs saline-treated animals). Mice treated with AuNS@CP nanoparticles showed more tumor inhibition than those treated with free GMP ( $p=0.02)$, which is consistent with EPR of drug-laden nanoparticles. [22, 23, 33] Tumor growth in the AuNS + laser and AuNS + GMP + laser groups were delayed compared to the saline + laser group (with a median tumor volume of $653 \mathrm{~mm}^{3}(\mathrm{p}=0.02)$ and $447 \mathrm{~mm}^{3}(\mathrm{p}=0.02)$ at day 15 , respectively). In the AuNS@CP + laser treated group, tumor growth was the slowest, with a median tumor volume of $138 \mathrm{~mm}^{3}$ on day 15 ( $\mathrm{p}=0.03$ vs AuNS@CP). These findings suggest that the combination of photothermal therapy and chemotherapy provided by AuNS@CP was more effective than either modality alone. On histologic analysis of hematoxylin-eosin stained sections of tumors, the cell density was much lower in the AuNS@CP + laser group than in the saline + laser group and AuNS@CP group (Fig. 5d, Fig S15), indicating that the combination of photothermal therapy and chemotherapy was more effective than chemotherapy alone.

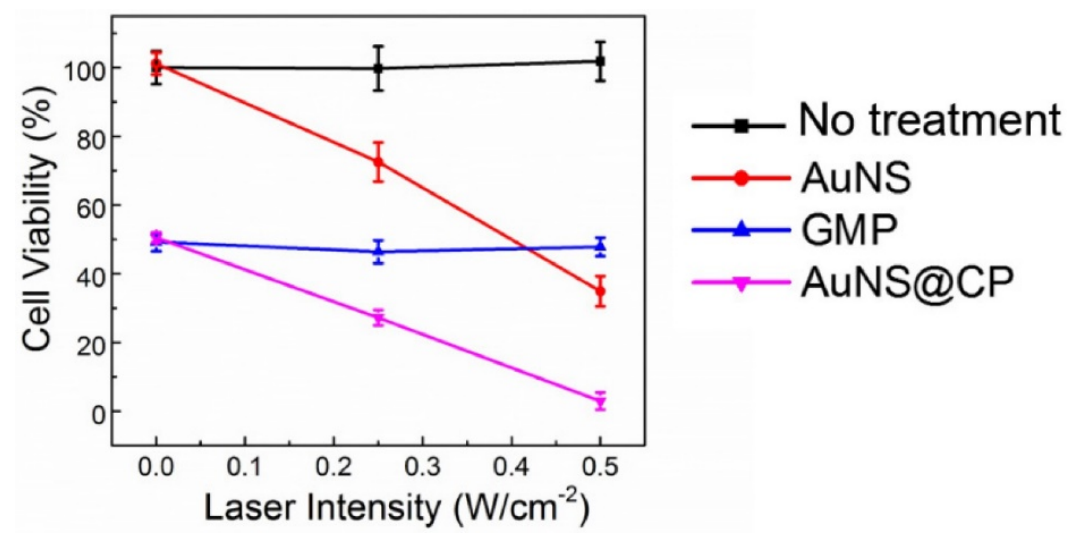

Figure 5. Cytotoxicity of AuNS, GMP, and AuNS@CP to 4T1 cells without or with irradiation. 4T1 cells were incubated with or without (no treatment) AuNS, GMP, or AuNS@CP (with an equivalent GMP concentration of $10 \mu \mathrm{g} / \mathrm{mL}$ and Au concentration of $11.7 \mu \mathrm{g} / \mathrm{mL}$ ) then irradiated with a $808 \mathrm{~nm}$ laser $(0,0.25$, or 0.5 $\mathrm{W} / \mathrm{cm}^{2}$ ) for $3 \mathrm{~min}, 6 \mathrm{~h}$ after exposure to treatment groups. After $24 \mathrm{~h}$, cell viability was quantitated by the MTS assay. Data are means \pm SD; $\mathrm{N}=4$. 

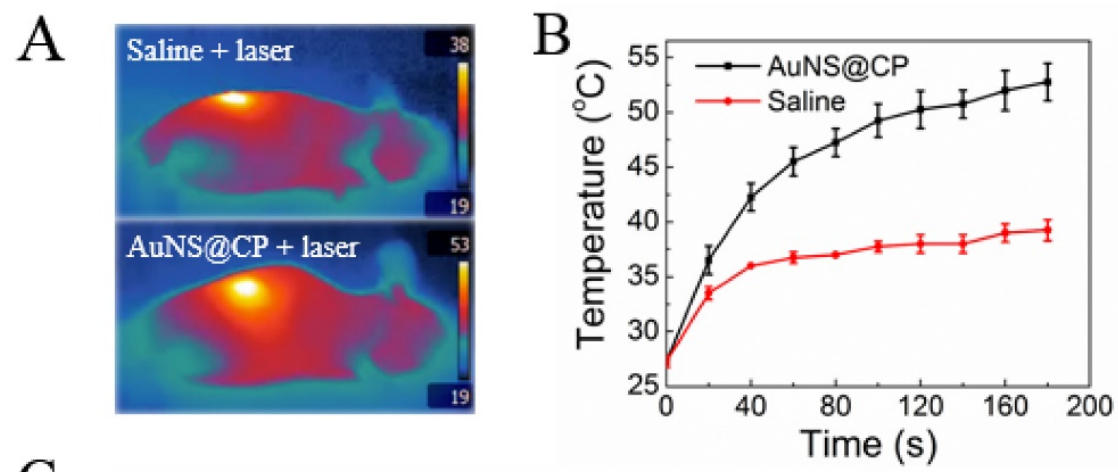

$\mathrm{C}$
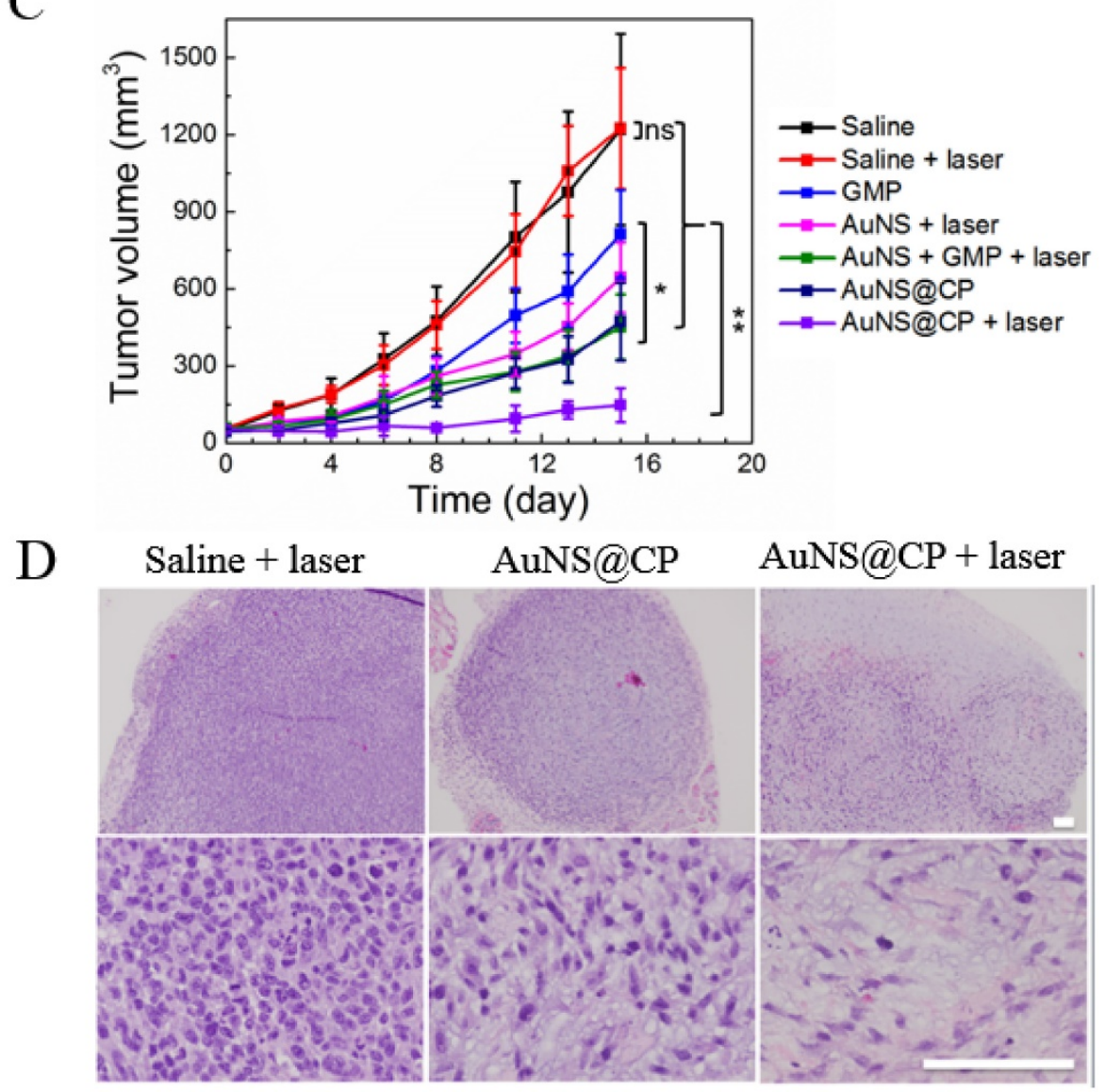

Figure 6. In vivo antitumor activity of AuNS@CP. (A) IR thermal images of $4 T 1$ tumor-bearing mice irradiated $\left(0.5 \mathrm{~W} / \mathrm{cm}^{2}, 3\right.$ min) 6 hours after intravenous administration of AuNS@CP nanoparticles or saline. (B) Time course of tumor temperature monitored by IR thermal camera during laser irradiation ( $0.5 \mathrm{~W} / \mathrm{cm}^{2}, 3$ $\min$ ) as in panel A, 6 hours after intravenous injection with AuNS@CP or saline. (C) Effect of various treatment groups on tumor volume. Data are means \pm SD ranges; $N=5$. $* P<0.05$, $* * P<0.01$. (D) Representative hematoxylin and eosin stained tumor sections collected from mice 3 days after treatment. Scale bar: $100 \mu m$.

The treatments appeared to have no detectable systemic toxicity. There was no change in average body weight in any of the experimental groups (Fig. S16). Histologic analysis of H\&E stained sections of various organs collected from the AuNS@CP + laser group on day 15 , the end of the experiment, did not show signs of organ damage or inflammatory lesions (Fig. S17).

\section{Conclusion}

Development of single-particle multifunctional platforms for theranostics remains a challenge. We have demonstrated a simple strategy for the integration of multiple therapeutic and imaging functionalities in AuNPs. The gold core of the AuNS@CP provided plasmonic photothermal effect and TPL, while the CP shell provided drug delivery and MR imaging, enabling multimodal imaging and combined photothermal and chemotherapy. MRI and TPL imaging could be used to monitor the in vivo distribution of AuNS@CP at the whole body and microscopic levels, respectively. 


\section{Supplementary Material}

Supplementary figures.

http://www.thno.org/v06p2306s1.pdf

\section{Acknowledgment}

We thank Dr. Scott E. Malstrom (Koch Institute) and Dr. Virginia Spanoudaki (MIT) for their assistance with MRI and data analysis and the histology core facility at Koch Institute for technical support. The work was supported by NIH Grant GM073626.

\section{Competing Interests} interest.

The authors declare no competing financial

\section{References}

1. Wang S, Huang $\mathrm{P}$, Chen XY. Stimuli-responsive programmed specific targeting in nanomedicine. ACS Nano. 2016; 10:2991-4

2. Maeda H, Nakamura H, Fang J. The EPR effect for macromolecular drug delivery to solid tumors: improvement of tumor uptake, lowering of systemic toxicity, and distinct tumor imaging in vivo. Adv Drug Deliv. 2013; 65: 71-9.

3. Tong $\mathrm{R}$, Chiang $\mathrm{HH}$, Kohane DS. Photoswitchable nanoparticles for in vivo cancer chemotherapy. Proc Natl Acad Sci USA. 2013; 110: 19048-53.

4. Prabhakar U, Maeda H, Jain RK, Sevick-Muraca EM, Zamboni W, Farokhzad $\mathrm{OC}$, et al. Challenges and key considerations of the enhanced permeability and retention effect for nanomedicine drug delivery in oncology. Cancer Res. 2013; 73: 2412-17.

5. Dong H, Du SR, Zheng XY, Lyu GM, Sun LD, Li LD, et al. Lanthanide nanoparticles: from design toward bioimaging and therapy. Chem Rev. 2015; 115: 10725-815

6. Xing H, Wong NY, Xiang Y, Lu Y. DNA aptamer functionalized nanomaterials for intracellular analysis, cancer cell imaging and drug delivery. Curr Opi in Chem Bio. 2012; 16: 429-35.

7. Liu J, Zheng X, Yan L, Zhou L, Tian G, Yin W, et al. Bismuth sulfide nanorods as a precision nanomedicine for in vivo multimodal imaging-guided photothermal therapy of tumor. ACS Nano. 2015; 9: 696-707.

8. Punjabi A, Wu X, Tokatli-Apollon A, El-Rifai M, Lee H, Zhang Y, et al. Amplifying the red-emission of upconverting nanoparticles for biocompatible clinically used prodrug-induced photodynamic therapy. ACS Nano. 2014; 8: 10621-30.

9. Qiu L, Chen T, Öçsoy I, Yasun E, Wu C, Zhu G, et al. A cell-targeted, size-photocontrollable, nuclear-uptake nanodrug delivery system for drug-resistant cancer therapy. Nano Lett. 2015; 15: 457-63.

10. Wang Z, Huang $\mathrm{P}$, Jacobson $\mathrm{O}$, Wang $\mathrm{Z}$, Liu $\mathrm{Y}$, Lin $\mathrm{L}$, et al. Biomineralization-inspired synthesis of copper sulfide-ferritin nanocages as cancer theranostics. ACS Nano. 2016; 10: 3453-60.

11. Dreaden EC, Mackey MA, Huang X, Kang B, El-Sayed MA. Beating cancer in multiple ways using nanogold. Chem Soc Rev. 2011; 40: 3391-04.

12. Dreaden EC, Alkilany AM, Huang X, Murphy CJ, El-Sayed MA. The golden age: gold nanoparticles for biomedicine. Chem Sov Rev. 2012; 41: 2740-79.

13. Yang X, Yang MX, Pang B, Vara M, Xia YN. Gold nanomaterials at work in biomedicine. Chem Rev. 2015; 115: 10410-88.

14. Huang P, Lin J, Li W, Rong P, Wang Z, Wang S, et al. Biodegradable gold nanovesicles with an ultrastrong plasmonic coupling effect for photoacoustic imaging and photothermal therapy. Angew Chem Int Ed. 2013; 52: 13958-64.

15. Cheng L, Wang C, Feng L, Yang K, Liu Z. Functional nanomaterials for phototherapies of cancer. Chem Rev. 2014; 114: 10869-939.

16. Zhang Z, Wang J, Chen C. Near-infrared light-mediated nanoplatforms for cancer thermo-chemotherapy and optical imaging. Adv Mater. 2013; 25: 3869-80.

17. Song T, Tang L, Tan LH, Wang X, Satyavolu NSR, Xing H, et al. DNA-encoded tuning of geometric and plasmonic properties of nanoparticles growing from gold nanorod seeds. Angew Chem Int Ed. 2015; 54: 8114-18.

18. Chen H, Zhang X, Dai S, Ma Y, Cui S, Achilefu S, et al. Multifunctional gold nanostar conjugates for tumor imaging and combined photothermal and chemo-therapy. Theranostics. 2013; 3: 633-49.

19. Espinosa A, Silva A, Sánchez-Iglesias A, Grzelczak M, Péchoux C, Desboeufs $\mathrm{K}$, et al. Cancer cell internalization of gold nanostars impacts their photothermal efficiency in vitro and in vivo: toward a plasmonic thermal fingerprint in tumoral environment. Adv Healthcare Mater. 2016; 5: 1040-8.

20. Yuan H, Fales AM, Vo-Dinh T. TAT peptide-functionalized gold nanostars: enhanced intracellular delivery and efficient NIR photothermal therapy using ultralow irradiance. J Am Chem Soc. 2012; 134: 11358-61
21. Wang S, Huang P, Nie L, Xing R, Liu D, Wang Z, et al. Single continuous wave laser induced photodynamic/plasmonic photothermal therapy using photosensitizer-functionalized gold nanostars. Adv Mater. 2013; 25: 3055-61.

22. Zhang Z, Wang L, Wang J, Jiang X, Li X, Hu Z., et al. Mesoporous silica-coated gold nanorods as a light-mediated multifunctional theranostic platform for cancer treatment. Adv Mater. 2012; 24: 1418-23.

23. Zhang Z, Wang J, Nie X, Wen T, Ji Y, Wu X, et al. Near infrared laser-induced targeted cancer therapy using thermoresponsive polymer encapsulated gold nanorods. J Am Chem Soc. 2014; 136: 7317-26.

24. Oh M, Mirkin CA. Chemically tailorable colloidal particles from infinite coordination polymers. Nature. 2005; 438: 651-54.

25. Horcajada P, Gref R, Baati T, Allan PK, Maurin G, Couvreur P, et al. Metal-organic frameworks in biomedicine. Chem Rev. 2012; 112: 1232-68.

26. Zhou HC, Long JR, Yaghi OM. Introduction to metal-organic frameworks. Chem Rev. 2012; 112: 673-74.

27. Rocca JD, Liu D, Lin WB. Nanoscale metal-organic frameworks for biomedical imaging and drug delivery. Acc. Chem. Res. 2011; 44: 957-68.

28. Carné A, Carbonell C, Imaz I, Maspoch D. Nanoscale metal-organic materials. Chem Soc Rev. 2011; 40: 291-305.

29. Spokoyny AM, Kim D, Sumreina A, Mirkin CA. Infinite coordination polymer nano- and microparticle structures. Chem Soc Rev. 2009; 38: 1218-27.

30. Li Y, Tang J, He L, Liu Y, Liu Y, Chen C, et al. Core-shell upconversion nanoparticle@metal-organic framework nanoprobes for luminescent/magnetic dual-mode targeted imaging. Adv Mater. 2015; 27: 4075-80.

31. Khaletskaya K, Reboul J, Meilikhov M, Nakahama M, Diring S, Tsujimoto M, et al. Integration of porous coordination polymers and gold nanorods into core-shell mesoscopic composites toward light-induced molecular release. J Am Chem Soc. 2013; 135: 10998-11005.

32. Papa AL, Sidiqui A, Balasubramanian SUA, Sarangi S, Luchette M, Sengupta $\mathrm{S}$, et al. PEGylated liposomal gemcitabine: insights into a potential breast cancer therapeutic. Cell Oncol. 2013; 36: 449-57.

33. Li L, Tong R, Li M, Kohane DS. Self-assembled gemcitabine-gadolinium nanoparticles for magnetic resonance imaging and cancer therapy. Acta Biomater. Acta Biomater. 2016; 33: 34-9.

34. Caravan P, Ellison J, McMurry TJ, Lauffer RB. Gadolinium(III) chelates as mri contrast agents: structure, dynamics, and applications. Chem Rev. 1999; 99: 2293-2352.

35. Xie JP, Lee JY, Wang DIC. Seedless, surfactantless, high-yield synthesis of branched gold nanocrystals in hepes buffer solution. Chem Mater. 2007; 19: 2823-30.

36. Patra CR, Bhattacharya R, Wang E, Katarya A, Lau JS, Dutta S,et al. Targeted delivery of gemcitabine to pancreatic adenocarcinoma using cetuximab as a targeting agent. Cancer Res. 2008; 68: 1970-78.

37. Zhao W, Gonzaga F, Li Y, Brook MA. Highly stabilized nucleotide-capped small gold nanoparticles with tunable size. Adv Mater. 2007; 19: 1766-71.

38. Hou Y, Qiao R, Fang F, Wang X, Dong C, Liu $\mathrm{K}$, et al. $\mathrm{NaGdF}_{4}$ nanoparticle-based molecular probes for magnetic resonance imaging of intraperitoneal tumor xenografts in vivo. ACS Nano. 2013; 7: 330-38.

39. Liu C, Gao Z, Zeng J, Hou Y, Fang F, Li Y, et al. Magnetic/Upconversion fluorescent NaGdF4:Yb, Er nanoparticle-based dual-modal molecular probes for imaging tiny tumors in vivo. ACS Nano. 2013; 7: 7227-40.

40. Boyd GT, Yu ZH, Shen YR. Photoinduced luminescence from the noble metals and its enhancement on roughened surfaces. Phy. Rev. B. 1986; 33: 7923-36.

41. Au L, Zhang $\mathrm{Q}$, Cobley CM, Gidding M, Schwartz AG, et al. Quantifying the cellular uptake of antibody-conjugated au nanocages by two-photon microscopy and inductively coupled plasma mass spectrometry. ACS Nano. 2010; 4: 35-42

42. Yuan H, Fales AM, Vo-Dinh T. TAT peptide-functionalized gold nanostars: enhanced intracellular delivery and efficient NIR photothermal therapy using ultralow irradiance. J Am Chem Soc. 2012; 134: 11358-61.

43. Zhang YS, Wang $Y$, Wang L, Wang $Y$, Cai X, Zhang $C$, et al. Labeling human mesenchymal stem cells with gold nanocages for in vitro and in vivo tracking by two-photon microscopy and photoacoustic microscopy. Theranostics 2013; 3: $532-43$

44. Dvorak HF, Nagy JA, Dvorak JT, Dvorak AM. Identification and characterization of the blood vessels of solid tumors that are leaky to circulating macromolecules. Am J Pathol 1988; 133: 95-109.

45. Jain RK, Munn LL, Fukumura D. Mammary Fat Pad Tumor Preparation in Mice. Cold Spring Harbor Protoc. 2012, 10: 1115-1116. 